\title{
Natural Vibration Frequencies of an SRF Module at Liquid-Helium Temperature
}

\author{
Ming-Hsun Tsai ${ }^{1,2, *}$, Lee-Long Han $^{1}$, Ming-Chyuan Lin $^{2}$, Chaoen Wang ${ }^{2}$, Meng-Shu Yeh ${ }^{2}$, Chih-Lung Lo $^{2}$, \\ Mei-Hsia Chang ${ }^{2}$ and Tsing-Tshih Tsung ${ }^{3}$ \\ ${ }^{1}$ Graduate Institute of Mechanical and Electrical Engineering, National Taipei University of Technology, Taipei, Taiwan \\ ${ }^{2}$ National Synchrotron Radiation Research Center, Hsinchu 30076, Taiwan \\ ${ }^{3}$ Department of Mechanical Engineering, Chinese Culture University, Taipei, Taiwan \\ *Corresponding author
}

\begin{abstract}
As a superconducting radio-frequency (SRF) cavity is a thin-shell structure with several thin bellows to bridge to its cryostat, any external excitation readily induces oscillations. The operation of a SRF module becomes unstable when the RF resonant frequency varies, due to mechanical vibrations, to a critical point. In this investigation we illustrate the mechanical vibration behavior of the 500-MHz SRF module of KEK type. An experimental test with an RF technique was used to assess the computational modes with models according to a finite-element method, for which material properties at various temperatures from $4.4 \mathrm{~K}$ to $300 \mathrm{~K}$ were assigned to the respective components. The calculated modes of vibration of each major component are shown to approach the computed results for the full structure, although some combined modes can be predicted only with the full model.
\end{abstract}

Keywords-cavity; finite-element method; measurement; superconducting; vibration

\section{INTRODUCTION}

The superconducting radio-frequency (SRF) module has become the major choice for a main accelerating cavity of synchrotron light sources. For instance, the CESR-type SRF module [1] is applied at the Canadian Light Source (CLS), Taiwan Light Source (TLS), Shanghai Synchrotron Radiation Facility (SSRF), Diamond Light Source (DLS), Pohang Light Source II (PLS-II) and National Synchrotron Light Source II (NSLS-II), whereas the KEK type [2] is operated in the collider super-KEKB [3], Beijing Electron-Positron Collider II (BECPII) [4] and soon in Taiwan Photon Source (TPS) [5]. Cavities of all these SRF modules have a thin shell structure and are located inside the liquid-helium vessel of the cryostat. Figure 1 shows the geometry and main dimensions of the 500-MHz SRF cavity of KEK type for TPS, with the outer conductor of a high-power input coupler (CPL), thermal transition sections of a small (SBP) and large (LBP) beam pipes, and the helium vessel shown together. Combined with the shell structures, thin bellows and the complicated cryostat, an SRF module has broad-band structural resonant modes, and thus vibrates readily on excitation with nearby external sources. An unstable operation can thus arise when the structural oscillation varies the RF resonant frequency of the cavity inside the cryostat to a critical point.

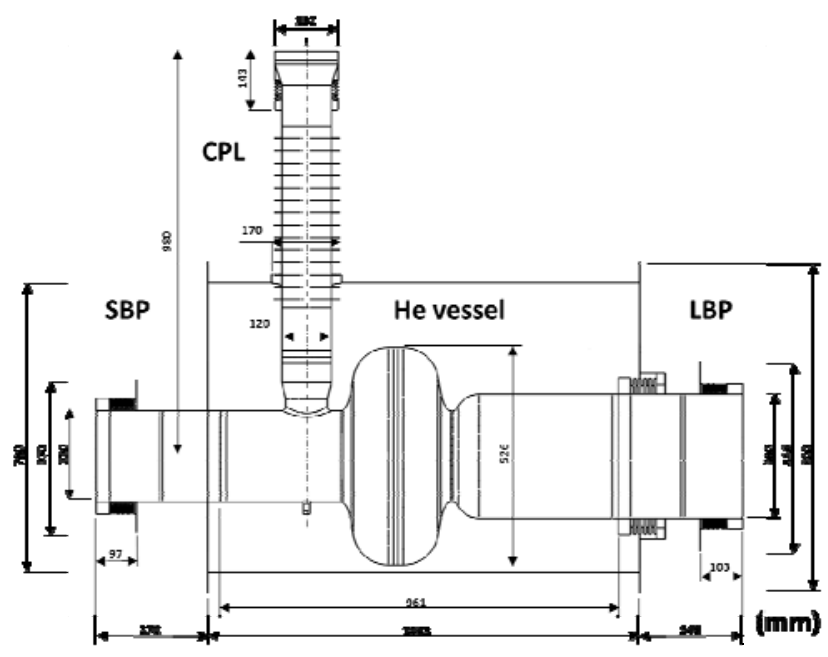

FIGURE I. GEOMETRY AND MAIN DIMENSIONS OF THE 500-MHz SRF CAVITY OF KEK TYPE, WITH OUTER CONDUCTOR OF THE COUPLER, THERMAL TRANSITION SECTIONS OF SMALL AND LARGE BEAM PIPES, AND THE HELIUM VESSEL SHOWN TOGETHER

For example, in TLS a superconducting (SC) wiggler is implemented downstream of the CESR-type SRF module; a transient thermal behavior of this SC wiggler during refilling with liquid helium invariably excites a mechanical oscillation of the SRF module and spontaneously varies its RF resonant frequency so as to modulate its RF matching condition. The conventional frequency tuning on deforming the SRF cavity cannot adequately compensate so fast a frequency change. Large variations of either transmitted or reverse RF power results in an occasional interruption of the machine operation. Illustrated in Fig. 2 are the signal histories during a trip event of the RF system of TLS, the cavity resonance frequency measured in terms of tuning angle starts to modulate in a frequency of about $20 \mathrm{~Hz}$ after being excited by longitudinal mechanical vibrations observed at the beam tube. This condition is intolerable against the demand for highly reliable SRF operation of a light source. Implementing a piezo-tuner of the SRF module provides a powerful means to correct actively the temporally varying RF resonant frequency of the SRF module, but the vibration mode of least frequency of the SRF module might constrain the applicable bandwidth of the piezo- 
tuner. Investigating the mechanical design of the SRF module to increase its smallest natural vibration frequency but to decrease the corresponding quality factor opens a possibility to minimize the troublesome operation resulting from issues of mechanical vibration.

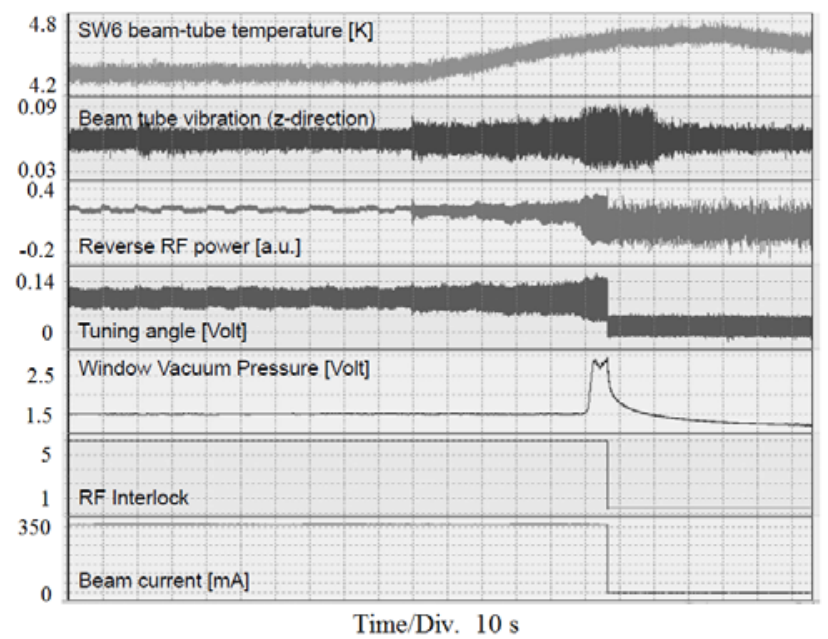

FIGURE II. EVENT CONSEQUENCE DURING A TRIP OF RF SYSTEM OF TLS

A commercially available code (SoldWorks Simulation [6]), based on the finite-element method that provides not only a function of modal analysis of structures [7] but also a transfer from CAD files to an analytical model, was selected for the analysis of the complicated mechanical model in this work. Several main components, including the cavity, the outer conductor of the coupler, thermal transition sections of small and large beam pipes and the helium vessel, were modeled to calculate their vibrational modes; a complete model to integrate all these components was established and analyzed to show the effects of integration on generating further modes. These computational results are compared to the results of measurements on a 500-MHz SRF module of KEK type for TPS. A detailed mechanism to introduce the structural resonant modes is thus realized, enabling further improvement to shift the modes to a greater frequency and to diminish the response sensitivity in future work.

\section{EXPERIMENTS}

Figure 3 shows the measurement setup on a 500-MHz SRF module of KEK type, in which a dynamic signal analyzer generated a signal at various frequencies to excite the piezotuner through a piezo-driver to amplify the signal; the phase detector measured the corresponding phase error between the forward RF power through the CPL and the transmitted RF power through an RF probe. To obtain the frequency response of the SRF module, the detected phase error was fed into a dynamic signal analyzer for comparison with the signal strength that it generated.

Figure 4 illustrates the measured result of the frequency response below $300 \mathrm{~Hz}$ of the $500-\mathrm{MHz}$ SRF module of KEK type for TPS, after being cooled with liquid helium to $4.4 \mathrm{~K}$.
Not only several major peaks, with values marked beginning at $74.16 \mathrm{~Hz}$, but also several small spikes appeared during the test. This SRF module hence has many resonant modes below 300 Hz. This test result then served for an examination with a numerical computation.

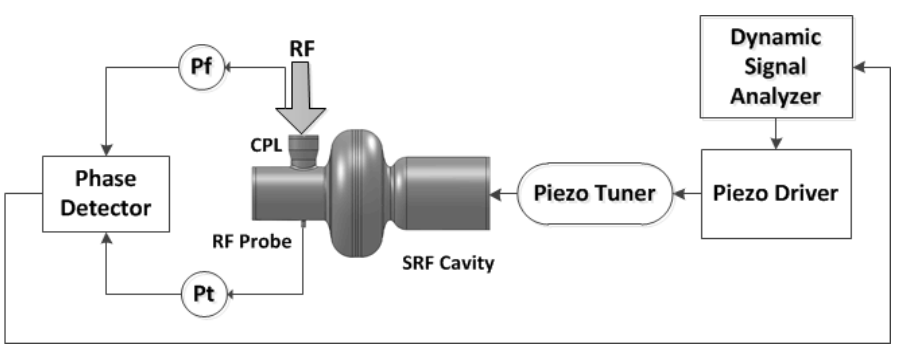

FIGURE III. EXPERIMENTAL SETUP FOR THE MEASUREMENT OF THE FREQUENCY RESPONSE OF THE 500-MHz SRF CAVITY OF KEK TYPE

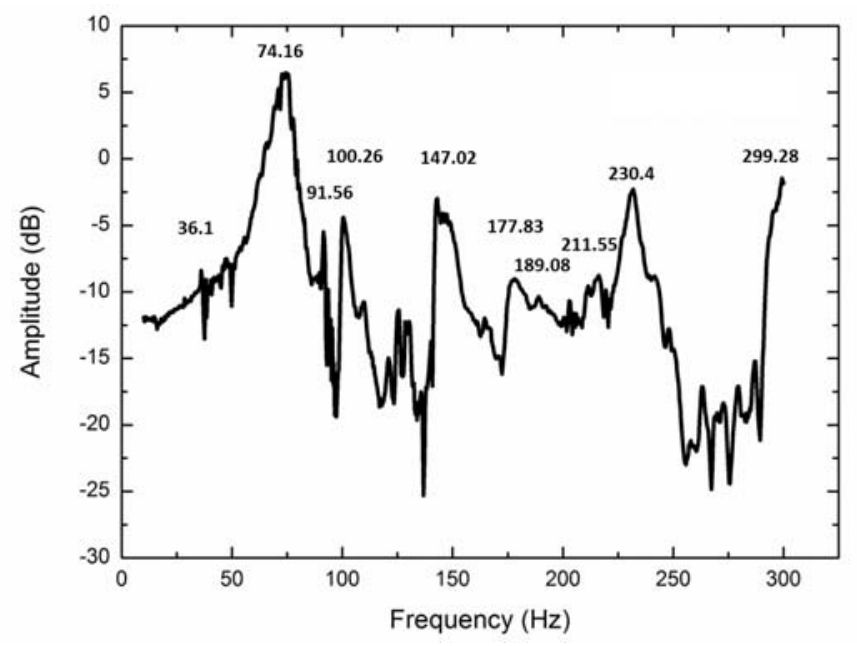

FIGURE IV. MEASURED FREQUENCY RESPONSE BELOW $300 \mathrm{~Hz}$ of the 500-MHz SRF MODULE OF KEK TYPE FOR TPS ON BEING COOLED TO $4.4 \mathrm{~K}$

\section{NUMERICAL MODEL}

A commercially available code (Solidworks Simulation [6]) was adopted to calculate the structural resonant frequencies of the KEK-type SRF module. To simplify the modeling and to decrease the computation resources, a triangular shell element was used to model the structure; appropriate boundary conditions were applied. Figure 5 shows the geometry and main dimensions of the bellows at various locations. These bellows are soft and thus contribute to the low-frequency modes to be discussed here. As the vacuum-shielding vessel is thick and strong, it is excluded from the model; all intersections with this vacuum-shielding vessel were instead given a fixed boundary condition. Another simplification was the absence of the structural damping, which has an influence less than $10 \%$ on the natural frequency of such a complicated structure. 


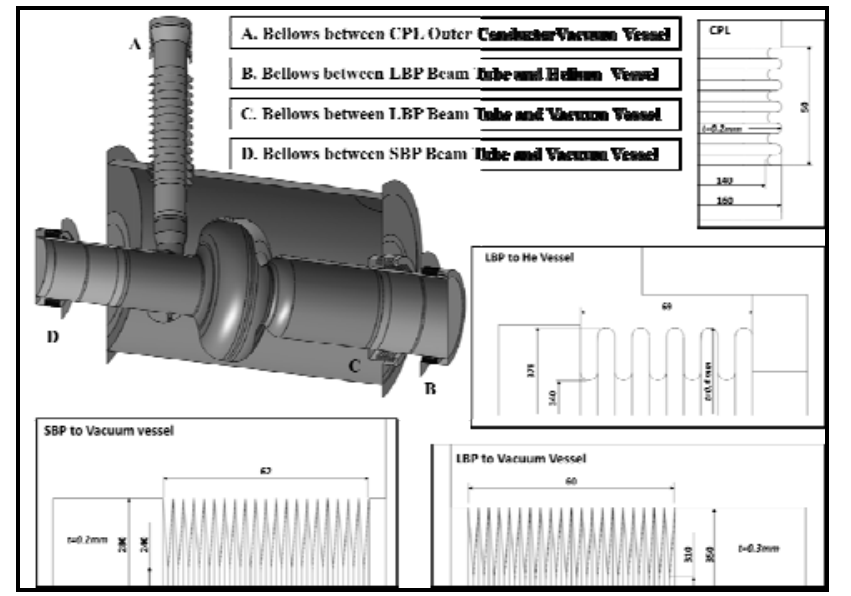

FIGURE V. GEOMETRY AND MAIN DIMENSIONS OF THE BELLOWS AT VARIOUS LOCATIONS OF THE 500-MHz SRF CAVITY OF KEK TYPE

The entire model has a wide temperature distribution, from $4.4 \mathrm{~K}$ for the part in contact with liquid helium, $80 \mathrm{~K}$ for the part in contact with liquid nitrogen, to $300 \mathrm{~K}$ for other parts in contact with the vacuum-shielding vessel. The corresponding material properties were assigned to the various components according to the material and the temperature. Table I lists the assigned Young's modulus $E$, Poisson's ratio $v$ and density $\rho$ of various materials - niobium for the cavity structure, stainless steel for the helium vessel, and copper for the outer conductor of the high-power coupler $[8,9]$.

TABLE I. TMECHANICAL PROPERTIES OF MATERIALS FOR SIMULATION

\begin{tabular}{|c|c|c|c|c|c|}
\hline & \multicolumn{3}{|c|}{$E / \mathrm{GPa}$} & \multirow[t]{2}{*}{$v$} & \multirow{2}{*}{$\frac{\rho}{/ \mathrm{kg} \mathrm{m}^{-3}}$} \\
\hline & $4.4 \mathrm{~K}$ & $77 \mathrm{~K}$ & $273 \mathrm{~K}$ & & \\
\hline $\mathrm{Nb}$ & 121 & 121 & 105 & 0.38 & 8570 \\
\hline SS 316 & 193 & 193 & 194 & 0.27 & 8000 \\
\hline $\mathrm{Cu}$ & 110 & 110 & 101 & 0.37 & 8900 \\
\hline
\end{tabular}

For comparison, some main components were individually modeled and computed to recognize their contribution to the vibrational modes. The appropriate material properties and boundary conditions were accordingly applied to these models. Much less computational resources were required, but some combined modes due to interactions with other components, such as variable flexibility and boundary conditions, were unavailable in any case.

\section{RESULTS AND DISCUSSION}

Table II lists the calculated natural vibrational frequencies of the full-module model and the individual components in the range below $300 \mathrm{~Hz}$. Each natural frequency of an individual component appears in the results of the full model, although a small difference might exist. The full model has, however, many more vibrational modes, because a combination of these components produces extra possibilities for natural vibration.
TABLE II. CALCULATED NATURAL VIBRATION FREQUENCY/HZ BELOW $300 \mathrm{~Hz}$

\begin{tabular}{|c|c|c|c|c|c|}
\hline Full module & SBP & LBP & CPL & Cavity & He Vessel \\
\hline 33.80 & 33.80 & & & & \\
\hline \multicolumn{6}{|l|}{38.50} \\
\hline \multicolumn{6}{|l|}{38.69} \\
\hline \multicolumn{6}{|l|}{40.72} \\
\hline 65.96 & 65.96 & & & & \\
\hline 66.37 & 66.37 & & & & \\
\hline 67.56 & 67.56 & & & & \\
\hline 79.24 & & 78.88 & & & \\
\hline \multicolumn{6}{|l|}{90.25} \\
\hline \multicolumn{6}{|l|}{91.17} \\
\hline 94.62 & 94.56 & & & & \\
\hline 95.17 & 95.11 & & & & \\
\hline 101.15 & 101.15 & & & & \\
\hline 132.96 & 132.96 & & & & \\
\hline 133.72 & 133.72 & & & & \\
\hline 134.64 & 134.64 & & & & \\
\hline 150.83 & 150.83 & & & & \\
\hline 151.07 & 151.07 & & & & \\
\hline 152.69 & 152.69 & & & & \\
\hline 152.88 & 152.88 & & & & \\
\hline 154.73 & & 154.69 & & & \\
\hline 156.22 & & 156.18 & 156.55 & & \\
\hline 157.49 & & 157.35 & 156.73 & & \\
\hline 161.65 & 161.64 & & & & \\
\hline 162.59 & 162.52 & & & & \\
\hline 167.78 & 167.78 & & & & \\
\hline 181.47 & 181.47 & & & & \\
\hline 181.64 & 181.64 & & & & \\
\hline \multicolumn{6}{|l|}{185.74} \\
\hline 186.69 & 186.69 & 186.36 & & & \\
\hline 186.94 & 186.94 & 187.23 & & & \\
\hline 194.67 & & 194.51 & & & \\
\hline 198.71 & 198.71 & & & & \\
\hline 199.70 & 199.70 & & & & \\
\hline \multicolumn{6}{|l|}{200.24} \\
\hline 200.80 & 200.80 & & & & \\
\hline \multicolumn{6}{|l|}{201.47} \\
\hline 212.22 & & 212.22 & & & \\
\hline 213.08 & & 213.09 & & & \\
\hline 214.84 & & 215.49 & & & \\
\hline 217.50 & 217.50 & & & & \\
\hline 217.68 & 217.67 & & & & \\
\hline 219.86 & 218.47 & & & & \\
\hline 224.97 & 221.09 & & & & \\
\hline 227.94 & & & & 227.38 & \\
\hline \multicolumn{6}{|l|}{229.57} \\
\hline \multicolumn{6}{|l|}{$\begin{array}{l}229.5 / \\
231.87\end{array}$} \\
\hline 231.95 & 231.95 & & & & \\
\hline 232.07 & 232.07 & & & & \\
\hline 233.34 & 233.25 & & & & \\
\hline 235.19 & 237.62 & & & & 237.42 \\
\hline 235.46 & 237.78 & & & & \\
\hline 255.43 & 242.89 & & & & \\
\hline 258.92 & 260.78 & & & & \\
\hline 262.47 & & & & & 260.55 \\
\hline 263.17 & 263.19 & & & & \\
\hline \multicolumn{6}{|l|}{263.63} \\
\hline \multicolumn{6}{|l|}{263.92} \\
\hline 264.25 & 264.24 & & & & \\
\hline 265.65 & 265.65 & & & & \\
\hline 265.75 & 265.72 & & & & \\
\hline 265.83 & 265.81 & & & 268.11 & \\
\hline 280.04 & & & & & \\
\hline 283.17 & 283.17 & & & & \\
\hline 283.34 & 283.34 & & & & \\
\hline 292.45 & 288.97 & & & & \\
\hline 296.68 & 294.29 & & & & \\
\hline 297.92 & 297.81 & & & & \\
\hline
\end{tabular}


Both these modes arise at their bellows to connect to the vacuum vessel, as components $\mathrm{C}$ and $\mathrm{D}$ shown in Fig. 5, and are along the longitudinal direction as Fig. 6 shows. These two vibrational frequencies are consistent with the measured results shown in Fig. 4 but slightly higher values, due to the structural damping was neglected in the numerical model. These two modes are important because that at $33.8 \mathrm{~Hz}$ is the first clear peak and that at $78.88 \mathrm{~Hz}$ represents the most intense peak in the measured frequency response; they are hence identified to arise at the bellows of the SBP and LBP; the bellows structure must thus improved. For example, these two bellows sections are welded bellows with great flexibility; replacements with formed bellows can greatly reinforce the structure, but the cost of the increased difficulty of assembling the module must be carefully examined.

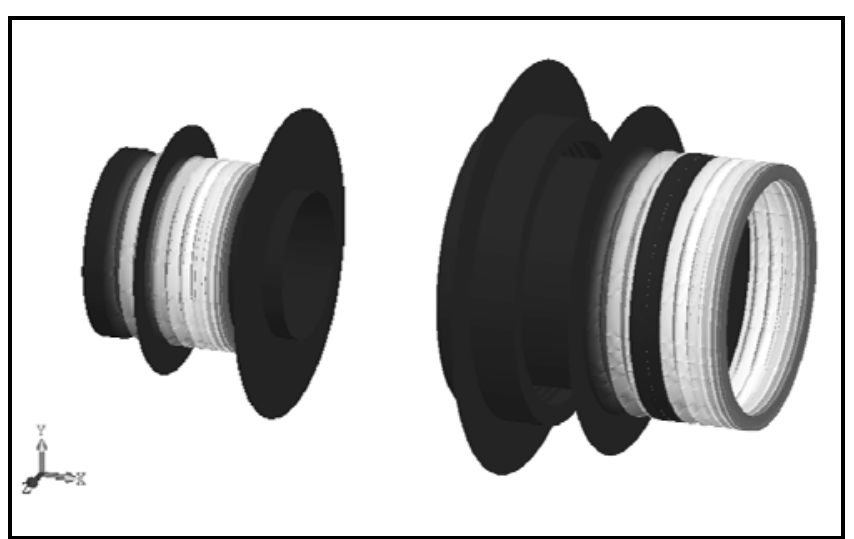

FIGURE VI. BOTH VIBRATIONAL MODES OF LEAST FREQUENCY AT THE SBP AND LBP ARISE AT THE BELLOWS CONNECTING TO THE VACUUM VESSEL, AND ARE ALONG THE LONGITUDINAL DIRECTION, WITH NATURAL FREQUENCIES 33.80 Hz AND 78.88 Hz, RESPECTIVELY

The outer conductor of the high-power input coupler has least natural frequency $156.55 \mathrm{~Hz}$, also arising at its bellows to the vacuum vessel as Fig. 7 shows. This frequency is near the second mode of the LBP. Both these modes are along the vertical direction and not discernible at the measured frequency response. In contrast, the third mode of the LBP behaves as a combined mode of the two bellows sections along the longitudinal direction, as Fig. 7 also shows; it appears again at the measured frequency response with peaks at $177.83 \mathrm{~Hz}$ and $189.08 \mathrm{~Hz}$. All these results hint strongly that the frequency response of this SRF module is more sensitive to the longitudinal vibration.

The calculated least vibrational frequencies of the cavity and the liquid-helium vessel are $227.38 \mathrm{~Hz}$ and $237.42 \mathrm{~Hz}$, respectively. Figure 8 shows these two modes together, near the weak locations with a hole. If necessary, reinforcements around the hole can greatly shift these two modes to much higher frequency, thus to eliminate their impacts.

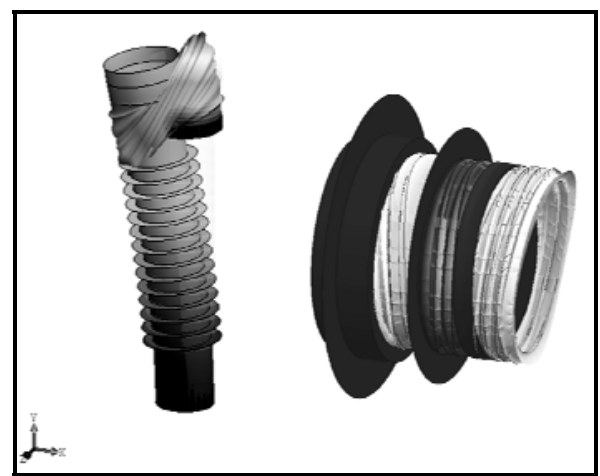

FIGURE VII. THE VIBRATIONAL MODE OF LEAST FREQUENCY FOR THE OUTER CONDUCTOR OF THE HIGH-POWER COUPLER ARISES AT ITS BELLOWS TO THE VACUUM VESSEL; THE THIRD MODE OF THE LARGE BEAM TUBE IS A COMBINATION FROM TWO BELLOWS SECTIONS

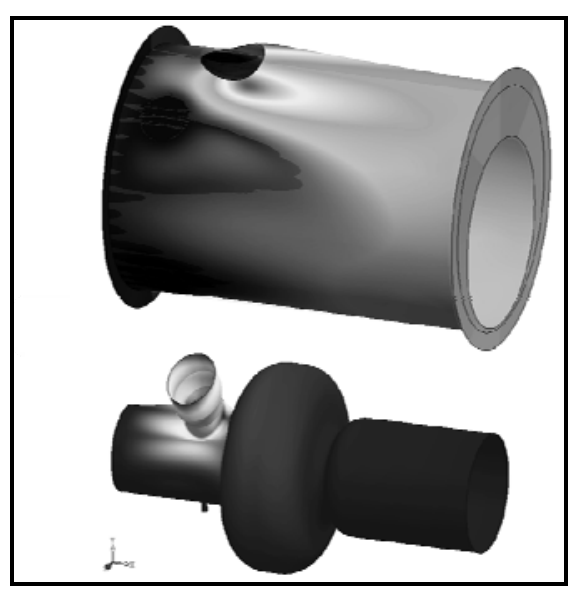

FIGURE VIII. VIBRATIONAL MODES OF LEAST FREQUENCY OF THE CAVITY AND THE LIQUID-HELIUM VESSEL, WITH NATURAL FREQUENCIES 227.38 AND 237.42 Hz, RESPECTIVELY CONCLUSION AND REMARK

\section{REFERENCES}

[1] H. Padamsee, P. Barnes, C. Chen, W. Hartung, J. Kirchgessner, D. Moffat, R. Ringrose, D. Rubin, Y. Samed, D. Saraniti, J. Sears, Q.S. Shu, and M. Tigner, "Design challenges for high-current storage rings," Part. Accel. Vol. 40, 1992, pp. 17-41.

[2] T. Furuya, et al., "A prototype module of a superconducting damped cavity for KEKB,” Proc. EPAC96, 1996, pp. 2121-2123.

[3] Y. Morita, et al., "Status of KEKB Superconducting Cavities and Study for Future SKEKB,” Proc. SRF2009, 2009, pp. 236-238.

[4] Y. Sun, et al., "Commissioning of BEPCII superconducting RF system," Proc. SRF2007, 2007, pp. 477-480.

[5] Ch. Wang, et al., "Design Features and Construction Progress of 500Mhz RF Systems for the Taiwan Photon Source,” Proc. PAC2011, 2011, pp.2513-2515.

[6] Dassault Systèmes SOLIDWORKS Corp., Waltham, MA, USA.

[7] P. Kurowski, "Engineering Analysis with SolidWorks Simulation 2012," Shroff Development Corp. Publications, Mission, Kansas, USA, 2012.

[8] J. Ekin, Experimental Techniques for Low-Temperature Measurements, Oxford University Press, Oxford UK, 2007.

[9] T. Flynn, Cryogenic Engineering, Marcel Dekker Inc., New York USA, 1997 